\title{
Power Doppler to investigate superficial digital flexor tendinopathy in the horse
}

\author{
Luca Lacitignola*, Santovito Rossella, De Luca Pasquale and Antonio Crovace \\ Dipartimento dell'Emergenza e dei Trapianti di Organi (D.E.T.O.), Sezione di Cliniche Veterinarie e P.A, Università \\ degli Studi di Bari “Aldo Moro”, s.p. per Casamassima Km 3, 70010 Valenzano, Italy
}

\begin{abstract}
Background: Recent advances in tendinopathy research have focused on tendon vascularization detected with Color Doppler (CD) ultrasound. The use of CD has also been described in horses in a study on chronic lesions of superficial digital flexor tendon and suspensory ligament.

Aim: The aim of this study was to investigate the occurrence and distribution of power Doppler (PD) signal in horses with superficial digital flexor tendinopathy.

Methods: Twenty-five horses with tendinopathy were included and 10 healthy horses with no lameness and no ultrasonographic abnormalities were used for comparison.

Results: In all horses with tendinopathy, the PD signal was visible while normal tendons with no abnormalities in B-mode were PD signal-free.

Conclusion: We can speculate that PD could provide essential information about the healing process than the grayscale ultrasound.

Keywords: Horse, Power Doppler, SDFT, Tendinopathy.
\end{abstract}

\section{Introduction}

Although there is still discussion about the role of neovascularization in tendinopathy, B-mode ultrasonography is routinely used for diagnosis of tendinopathy and desmopathy both in human and equine species. Recent advances in tendinopathy research have focused on tendon vascularization detected with color Doppler (CD) ultrasound (Terslev et al., 2001). In a study on the midportion of the Achilles' tendon of human beings, CD ultrasound revealed vascularization in the painful area with structural changes in tendons with chronic tendinopathy but not in pain-free normal tendons (Ohberg et al., 2001). The use of CD has also been described in horses in a study on chronic lesions of superficial digital flexor tendon (SDFT) and suspensory ligament, which confirmed the absence of CD in normal tendons and ligaments (Kristoffersen et al., 2005). CD signal was in contrary detected in affected SDFT and suspensory ligament (Kristoffersen et al., 2005).

More recently, other authors stated that blood flows appearing on CD images of equine SDFT were indicative of vessels in residual inflammatory granulation tissue replenishing the injured tendon, concluding that $\mathrm{CD}$ images could provide another ultrasonographic method for differentiating between inflammatory granulation and hypovascular scar tissues during the remodeling phase of tendon healing (Murata et al., 2012).

Power Doppler (PD), unlike CD, is independent of the probe being aligned to the flow and can detect abnormal vascularity in tendons with higher sensitivity compared to CD (Richards et al., 2005). PD ultrasound has demonstrated to detect blood flow in Achilles' tendinopathy even when the vessels are too small to be visualized with spectral ultrasound or magnetic resonance imaging (MRI) (Richards et al., 2010). PD ultrasound has recently been used in the horse to assess the vascularity of suspensory branches in horses (Rabba et al., 2018).

For the assessment of the severity of neovascularization, scoring systems such as the modified Ohberg et al. (2001) or Filippucci-Grassi scoring system (Filippucci et al., 2007) have been reliably used to estimate the Doppler signal in clinical studies (Rabba et al., 2018). The aims of this study were to investigate the occurrence of tendon vascularity by means of PD and to compare B-mode to PD ultrasonographic images of SDFTs in horses with tendinopathy of digital flexor tendons.

\section{Materials and Methods}

\section{Horses-inclusion criteria}

Lame horses (group L) were prospectively included if they were diagnosed to have superficial digital flexor tendinopathy based on the B-mode ultrasonographic examination that occurred in 3 weeks since lameness onset. The severity of lameness at the trot was graded from 1 to 5 on a scale of 5 [American Association of Equine Practitioners (AAEP) scoring system]. Regional analgesia was used to confirm the source of pain, depending on the visibility and/or severity of clinical signs. Ten non-lame horses, with neither

*Corresponding Author: Luca Lacitignola. D.E.T.O., sezione di Cliniche Veterinarie e P.A, Università degli Studi di Bari “Aldo Moro", s.p. per Casamassima Km 3, 70010. Valenzano, BARI, Italy. Email: luca.lacitignola@uniba.it 
clinical nor ultrasonographic signs of tendinopathy of digital flexor tendons, were used as a control group (group C).

\section{Ultrasonographic examination-material and technique}

All ultrasonography were performed by the same operator. Ultrasound examination in group L was performed at least 6 hours after diagnostic analgesia. Horses were sedated with detomidine chlorohydrate 10 $\mathrm{mcg} / \mathrm{kg}$. The hair was clipped, the skin was degreased with alcohol or warm water, and the acoustic gel was applied. The tendons were examined with a linear transducer (frequency used ranging from 7.5 to 12 $\mathrm{MHz}$ ), and a stand-off pad was used. Different portable and non-portable ultrasound machines were used for the study (GE PRO SERIES LOGIC 400, MEDISON SONOVET R3, ESAOTE Mylab 30). B-mode longitudinal and transverse sections were realized on the weight-bearing limb. Non-weight-bearing and dynamic examinations were realized when necessary to examine the manica flexoria and relative flexor tendon sliding in B-mode. Machine setting for PD evaluation required to set for low flow with the lowest possible pulse repetition frequency and the lowest possible wall filter. The color gain was set just below the noise level. The focus was placed where the highest sensitivity was required or just below the region of interest. The color box was large and placed on the entire section area of SDFT. For the PD examination, the limb was flexed to relax the tendons. The structures were also examined in longitudinal and transverse planes without a stand-off pad.

\section{$B$-mode and PD scoring}

Ultrasonographic images were saved in Digital Imaging and Communications in Medicine (DICOM) format for off-line review; in the control group of SDFT, crosssectional area (CSA) and the PD score assignment were measured at zones 2A. In group L, CSA and Scoring assignment were performed at maximum injury zone. In B-mode, images were scored using Type Lesion Echogenicity Score (ES) attributed as 0 (isoechoic), -1 (hypoechoic), -2 (more hypoechoic), -3 (anechoic), +1 (hyperechoic), and +2 (more hyperechoic). Fiber Pattern Score (FPS) was considered as alignment of collagen fibers in longitudinal scans; low values indicated normal pattern, while higher values were related to completely disrupted pattern: $>75 \% 0 ; 50 \%-$ $75 \% 1 ; 25 \%-50 \% 2$; and $<25 \% 3$.

PD signal was scored using two different scores: Filippucci-Grassi semi-quantitative scoring system [Power Doppler Filippucci-Grassi (PDFG)] (Filippucci et al., 2007) (grade 0: no flow; grade 1: single vessel signals; grade 2: $<50 \%$ of lesion area filled with vessels; and grade $3: \geq 50 \%$ lesion area filled with vessels) and modified Ohberg score [Power Doppler Ohberg score (PDOH)] (Ohberg et al., 2001) (grade 0 : no flow; grade 1: one vessel throughout the tendon; grade 2: two vessels throughout the tendon; grade 3: three vessels throughout the tendon; grade $4:>$ three vessels throughout the tendon). B-mode and PD scores were assigned by the same reader.

\section{Statistical analysis}

Data were analyzed by Minitab® 15.1.1.0. Software (LEAD Technologies, Inc). Analysis of Spearman's rank correlation coefficient was performed among B-mode and PD scores. Significance level has been fixed at $p<0.05$.

\section{Ethical approval}

The study involved clinical cases. No experimental procedures have been executed, the PD examination is a standard non-invasive technique currently used, as $\mathrm{CD}$, in addition to the standard B-mode, to study blood flux. Animal's owners were informed and gave permission. In compliance with National animal care legislation, the Institutional ethics committee of the Department of Emergencies and Organ Transplantation of the University of Bari approved this study.

\section{Results}

\section{Lame horses (group L) and clinical data}

Twenty-five horses corresponded to selection criteria. Horses included were mainly Standardbred (13 Standardbreds, 2 Angloarabs, 2 Haflinger, 2 ponies, 2 quarter horses, 3 Italian saddle horses, and 1 warmblood), aged $2-23$ years (mean age $8.32 \pm 4.84$ years old and were $400-650 \mathrm{~kg}$ (mean $484.99 \pm 67.34 \mathrm{~kg}$ ) in weight. Twenty-two affected horses had a unilateral forelimb and three horses had hindlimb lameness. Degree of lameness was meanly $2.68 \pm 0.48$. The SDFT lesions were located in the metacarpal and metatarsal region.

\section{B-mode ultrasonographic examination}

Tendons of horses of group $\mathrm{C}$ had homogeneous echogenicity and regular fiber pattern with B-mode scores (ES and FPS) equal to 0. Mean CSA was 0.76 $\pm 0.05 \mathrm{~cm}^{2}$. In group L, CSA increased significantly versus control group $(p<0.01)$, measuring meanly 1.74 $\pm 0.66 \mathrm{~cm}^{2}$. Mean ES of the ill-defined areas with the SDFT was $2.00 \pm 0.82$ and the mean fiber pattern score (FPS) was $2.32 \pm 0.69$.

\section{PD ultrasonographic examination}

No intratendinous PD signal was detected (PD scores equal to 0 ) in tendons of horses of group $C$; only positive PD signal was detectable in intertendinous space (Fig. 1).

All tendon lesions of horses of group L showed intratendinous vascularization. Mean PD signal of the illdefined area within the SDFT was $2.48 \pm 0.65$ with the PDFG scale, while $3.4 \pm 0.82$ with PDOH scale (Fig. 2). Correlation among $B$-mode scores and PD scores

No correlation was observed among ES and PD score neither using the Filippucci-Grassi scoring system nor using the Ohemberg. Also, FPS was not correlated with PDFG nor PDOH. Also, CSA was not correlated to any examined scoring system.

Correlation between the PD scores calculated based on the two scoring systems was very high (Spearman's $r=0.95, p<0.05$ ). 




Fig. 1. No intratendinous PD signal was detected (PD scores equal to 0) in tendons of horses of group C; only positive PD signal was detectable in interfascicular space. The figure shows typical PD signaling in the normal tendon.

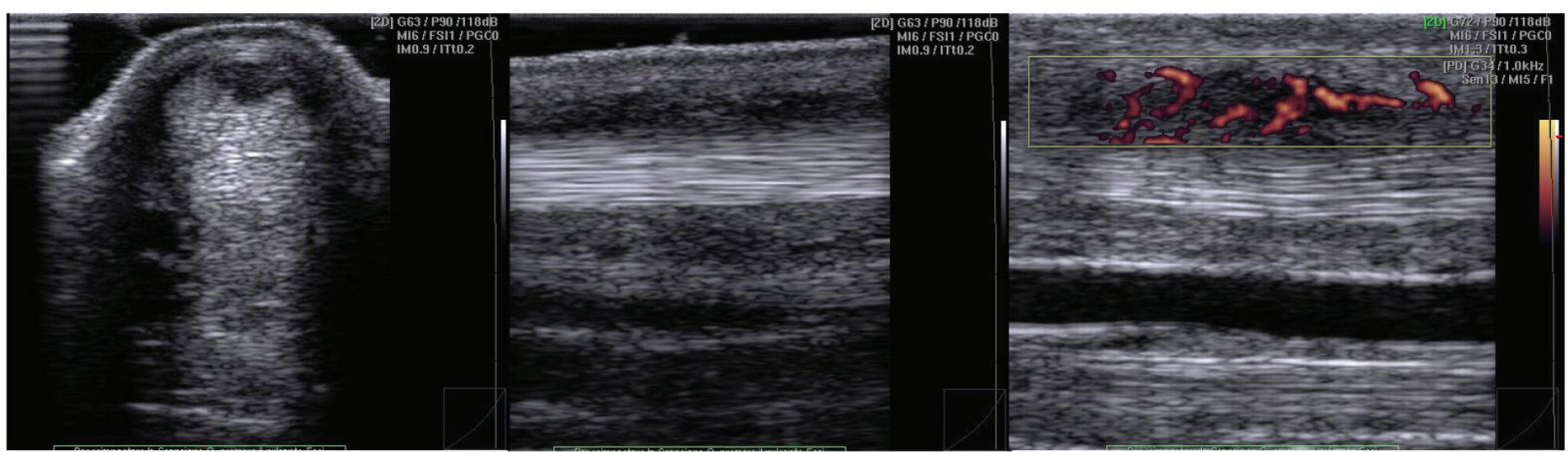

Fig. 2. Representative US images of an injured tendon showing intratendinous vascularization. PD signal has been scored in FG 3.

\section{Discussion}

The exact role of neovascularization or angioblastic hyperplasia in tendinopathy remains an issue of discussion; it has been suggested that the invasion and proliferation of new blood vessels may be a contributory factor to the pain and chronicity of the disease (Peers et al., 2003). In equine species, increased vascularity has been recently introduced as one of the histological parameters to define tendinopathy, for differentiating between inflammatory granulation and hypovascular scar tissues during the remodeling phase of tendon healing (Murata et al., 2012; Smith and McIlwraith, 2012). In this study, we investigate normal and injured SDFT by the use of PD to assess the blood flow within the SDFT.

In the normal tendon, the structure is not possible to verify the presence of PD signal within the tendon tissue, but only in the interfascicular zone (Fig. 1). Confirming that in horses, normal tendon shows only sparse vasculature in the interfascicular zone (Smith and McIlwraith, 2012) and verified, both in equine and in human beings species, with the use of CD (de Vos et al., 2007; Kristoffersen et al., 2005) and also by histopathological investigations (Murata et al., 2012). In our study, tendons with clinical and ultrasonographic signs of tendinopathy (group L), have an increased PD score and PD signal is not only visible in the interfascicular zone but also within the tendon lesion (intratendinous zone) (Fig. 2). A validated neovascularization score is essential in the evaluation of tendinopathy, and future studies can be focused on the interobserver and intraobserver reliability.

In the studies on neovascularization in human beings, several neovascularization scoring systems are used. Some authors make a distinction between the presence or absence of neovascularization (Alfredson and Ohberg, 2005; Zanetti et al., 2003) while other authors used a grading system from 0 to $4+$ (Boesen et al., 2006a; Lind et al., 2006; Ohberg and Alfredson, 2002) or from 0 to $3+$ (Reiter et al., 2004). Two 
studies described the use of color fraction or surface measurement of colored pixels (Boesen et al., 2006b; Peers et al., 2003). In the horse medicine field, the CD pulsating signal is scored in equine SDF tendinopathy as Grade 1, rhythmically blinking Grade 2, pulsatile; Grade 3 streaming; this scoring system applied the corresponding histopathologically with the increase of color activity (Murata et al., 2012). In equine medicine, a dedicated scale has never been described.

In our study, the use of a dedicated PD scoring system as PDFG or PDOH is simple and easy to use in daily clinical practice, moreover, allows the score assignment after performing the examination. Both scoring systems in this study showed no statistical differences, so we can state that both scores could be equally used in the equine species.

No correlation has been found about ES, nor CSA, and both PDFG and PDOH. ES, usually employed as severity parameter and time of occurred injury, is not an indicator of neovessels invasion during healing. Indeed, neovascularization, CD or PD detected, is frequently thought to seem to be associated with pain of Achilles tendinopathy (de Vos et al., 2007; Ohberg et al., 2001; Rabba et al., 2018). Hypoechoic or anechoic lesions in equine tendons have been evaluated usually in the acute phase of tendinopathy, as result of hemorrhagic phase or during the formation of granulation tissue that occurs in 2 weeks after injury (Sharma and Maffulli, 2005), but no information about blood flow can be given only with the grayscale US. We can speculate that PD could provide essential information about the stage of healing than the grayscale US, as previously stated for CD (Murata et al., 2012), to detect active granulation tissue (vascularized) instead of scar tissue (no vascularized). This information could equip the clinician with essential information to improve the timing of treating tendon injuries with stem cell therapy. It is important to allow adequate angiogenesis and granulation tissue to support engrafted cells, then an earlier hemorrhagic lesion (Smith et al., 2003).

Although the PD is a new technique for diagnostic imaging of tendinopathy in horses, it is much more sensitive than grayscale ultrasound or $\mathrm{CD}$ to visualize the vasculature that with the other two methods would not be manifested, some important limits are to be evaluated. One of the first goals is standardization of the technique for evaluations of tendon injuries of the horses. Also, a proper limb flexion and patient restraint are essential to minimize the movements that interfere with visualization of the vessels with the $\mathrm{PD}$, resulting in false-positive signals. A recent study stated that PD signal being more evident in suspensory ligament branches with more severe B-mode changes in lame horses (Rabba et al., 2018). In accordance with this, author longitudinal studies based on larger caseload are needed to understand the mechanism responsible for
PD signal and to correlate increased blood flow with the stage of the lesion (Rabba et al., 2018).

In conclusion, we can suggest that the $\mathrm{PD}$ is a promising diagnostic aid in the evaluation of equine tendon injuries, providing a wide range of comprehensive information concerning the neovascularization of SDFT lesions, but monitoring of healing of injured tendons and prognosis detecting neovascularization is still controversial.

\section{Authors' contribution}

Luca Lacitignola: Study design, statistical analysis, and writing of the manuscript. Santovito Rossella: Data collection. De Luca Pasquale: Data collection. Antonio Crovace: Supervision.

\section{References}

Alfredson, H. and Ohberg, L. 2005. Neovascularisation in chronic painful patellar tendinosis - promising results after sclerosing neovessels outside the tendon challenge the need for surgery. Knee Surg. Sports Traumatol. Arthrosc. 13, 74-80.

Boesen, M.I., Boesen, A., Koenig, M.J., Bliddal, H. and Torp-Pedersen, S. 2006a. Ultrasonographic investigation of the Achilles tendon in elite badminton players using color Doppler. Am. J. Sports Med. 34, 2013-2021.

Boesen, M.I., Koenig, M.J., Torp-Pedersen, S., Bliddal, H. and Langberg, H. 2006b. Tendinopathy and Doppler activity: the vascular response of the Achilles tendon to exercise. Scand. J. Med. Sci. Sports 16, 463-469.

de Vos, R.J., Weir, A., Cobben, L.P. and Tol, J.L. 2007. The value of power Doppler ultrasonography in Achilles tendinopathy: a prospective study. Am. J. Sports Med. 35, 1696-1701.

Filippucci, E., Meenagh, G., Delle Sedie, A., Riente, L., Iagnocco, A., Bombardieri, S., Valesini, G. and Grassi, W. 2007. Ultrasound imaging for the rheumatologist XII. Ultrasound imaging in sports medicine. Clin. Exp. Rheumatol. 25, 806-809.

Kristoffersen, M., Ohberg, L., Johnston, C. and Alfredson, H. 2005. Neovascularisation in chronic tendon injuries detected with color Doppler ultrasound in horse and man: implications for research and treatment. Knee Surg. Sports Traumatol. Arthrosc. 13, 505-508.

Lind, B., Ohberg, L. and Alfredson, H. 2006. Sclerosing polidocanol injections in mid-portion Achilles tendinosis: remaining good clinical results and decreased tendon thickness at 2-year followup. Knee Surg. Sports Traumatol. Arthrosc. 14, $1327-1332$.

Murata, D., Misumi, K. and Fujiki, M. 2012. A preliminary study of diagnostic color Doppler ultrasonography in equine superficial digital flexor tendonitis. J. Vet. Med. Sci. 74, 1639-1642. 
Ohberg, L. and Alfredson, H. 2002. Ultrasound guided sclerosis of neovessels in painful chronic Achilles tendinosis: pilot study of a new treatment. Br. J. Sports Med. 36, 173-177.

Ohberg, L., Lorentzon, R. and Alfredson, H. 2001. Neovascularisation in Achilles tendons with painful tendinosis but not in normal tendons: an ultrasonographic investigation. Knee Surg. Sports Traumatol. Arthrosc. 9, 233-238.

Peers, K.H., Brys, P.P. and Lysens, R.J. 2003. Correlation between power Doppler ultrasonography and clinical severity in Achilles tendinopathy. Int. Orthop. 27, 180-183.

Rabba, S., Grulke, S., Verwilghen, D., Evrard, L. and Busoni, V. 2018. B-mode and power Doppler ultrasonography of the equine suspensory ligament branches: a descriptive study on 13 horses. Vet. Radiol. Ultrasound 59, 453-460.

Reiter, M., Ulreich, N., Dirisamer, A., Tscholakoff, D. and Bucek, R.A. 2004. Color and power Doppler sonography in symptomatic Achilles tendon disease. Int. J. Sports Med. 25, 301-305.

Richards, P.J., McCall, I.W., Day, C., Belcher, J. and Maffulli, N. 2010. Longitudinal microvascularity in Achilles tendinopathy (power Doppler ultrasound, magnetic resonance imaging time-intensity curves and the Victorian Institute of Sport Assessment-
Achilles questionnaire): a pilot study. Skeletal Radiol. 39, 509-521.

Richards, P.J., Win, T. and Jones, P.W. 2005. The distribution of microvascular response in Achilles tendonopathy assessed by color and power Doppler. Skeletal Radiol. 34, 336-342.

Sharma, P. and Maffulli, N. 2005. Tendon injury and tendinopathy: healing and repair. J. Bone Joint Surg. Am. 87, 187-202.

Smith, R.K. and McIlwraith, C.W. 2012. Consensus on equine tendon disease: building on the 2007 Havemeyer symposium. Equine Vet. J. 44, 2-6.

Smith, R.K., Korda, M., Blunn, G.W. and Goodship, A.E. 2003. Isolation and implantation of autologous equine mesenchymal stem cells from bone marrow into the superficial digital flexor tendon as a potential novel treatment. Equine Vet. J. 35, 99-102.

Terslev, L., Qvistgaard, E., Torp-Pedersen, S., Laetgaard, J., Danneskiold-Samsoe, B. and Bliddal, H. 2001. Ultrasound and power Doppler findings in jumper's knee-preliminary observations. Eur. J. Ultrasound 13, 183-189.

Zanetti, M., Metzdork, A., Kendert, H.P., Zollinger, H., Vienne, P. and Siefert, B. 2003. Achilles tendons: clinical relevance of neovascularisation diagnosed with power Doppler US. Radiology 227, 556-560. 